\title{
Finding the Optimal Compression Level for Strain-Encoded (SENC) Breast MRI; Simulations and Phantom Experiments
}

\author{
Ahmed A. Harouni ${ }^{1}$, Michael A. Jacobs ${ }^{2}$, and Nael F. Osman ${ }^{1,2}$ \\ ${ }^{1}$ Electrical and Computer Engineering, Johns Hopkins University \\ harouni@jhu.edu \\ ${ }^{2}$ Russell H. Morgan Department of Radiology and Oncology, Johns Hopkins University school \\ of medicine, Baltimore, MD, USA
}

\begin{abstract}
Breast cancer is the most common cancer among women and the second highest cause of cancer-related death. Diagnostic magnetic resonance imaging (MRI) is recommended to screen high-risk patients. Strain-Encoded (SENC) can improve MRI's specificity by detecting and differentiating masses according to their stiffness. Previous phantom and ex-vivo studies have utilized SENC to detect cancerous masses. However, SENC required a $30 \%$ compression of the tissue, which may not be feasible for in-vivo imaging. In this work, we use finite element method simulations and phantom experiments to determine the minimum compression required to detect and classify masses. Results show that SENC is capable of detecting stiff masses at compression level of 7\%, though higher compression is needed in order to differentiate between normal tissue and benign or malignant masses. With on-line SENC calculations implemented on the scanner console, we propose to start with small compressions for maximum patient comfort, then progress to larger compressions if any masses are detected.
\end{abstract}

\section{Introduction}

According to the American Cancer Society's 2009 report [1], one in eight women will develop breast cancer in her lifetime. Early detection of breast lesions using mammography has resulted in lower mortality rates. However, some breast lesions are mammography occult (e.g. dense breasts) and the use of magnetic resonance imaging (MRI) is recommended, especially for women who are at high risk of developing breast cancer.

MRI has high sensitivity (95\%) and moderate specificity (83\%) [2]. Cancerous masses are 3-13 times stiffer than normal tissue [3]; therefore, MRI's Specificity can be increased by incorporating the tissue's stiffness. Osman et al. developed StrainEncoded (SENC) MRI [4] to directly measures strain, which is inversely proportional to stiffness. The feasibility of SENC to detect stiff masses in a homogeneous phantom was shown in [5]6] using a simple hardware with single compression that limited the scan time to one second and the scanning resolution to $4 \times 4 \times 10 \mathrm{~mm}^{3}$. In [7], we introduced a new hardware capable of accurately repeating compressions, which allows to increase scanning time. Thus, achieving a high resolution of $1 \times 1 \times 5 \mathrm{~mm}^{3}$ and significantly improving signal-to-noise ratio and contrast-to-noise ratio. Then in a later

G. Fichtinger, A. Martel, and T. Peters (Eds.): MICCAI 2011, Part I, LNCS 6891, pp. 444 451, 2011.

(C) Springer-Verlag Berlin Heidelberg 2011 
study [8], an alternation of SENC called SENC relaxation (SENC-REX) was introduced to complement traditional SENC compression (SENC-CMP). Phantom and ex-vivo results showed the potential of SENC-CMP and SENC-REX to detect cancerous masses. Currently however, SENC requires multiple compressions of 30\%. This compression level was originally developed and optimized to image the myocardial circumferential strain, which ranges from $+5 \%$ to $-30 \%$ [4], but such a high compression may prove to be uncomfortable or infeasible in a clinical setting.

In this work, we focus on determining the minimum amount of compression necessary to detect and differentiate between benign and malignant masses. We built a phantom that contains masses with known stiffness, performed finite element method simulations and compared the results with acquired MRI data.

\section{Methods and Material}

\subsection{Hardware}

We used our hardware previously described in [7] for compression. The hardware consists of two air-cylinders fitted underneath a standard MR breast coil. Figure 1 a shows a patient laying in prone position with two air-cylinders compressing each breast. Figure $1 \mathrm{~b}$ shows a top-view of a single side of the hardware compressing a breast phantom. The hardware can operate in either compression or relaxation mode, generating SENC-CMP and SENC-REX strain images, which examine the compression and relaxation properties of the tissue, respectively. More details can be found in [78].
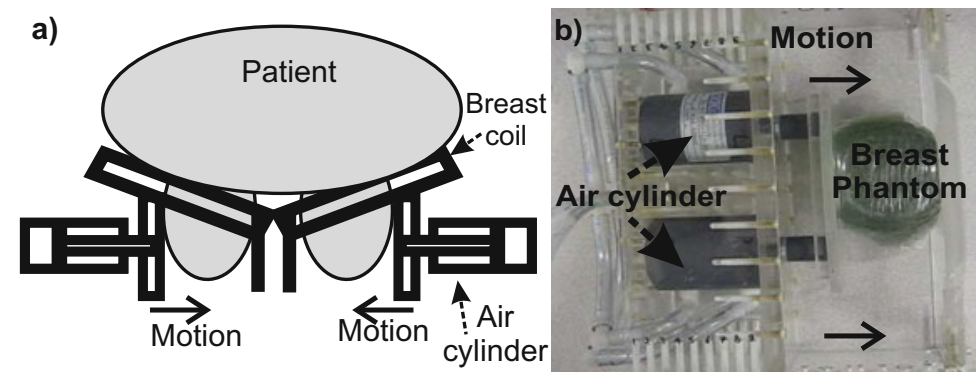

Fig. 1. a) Schematic of the hardware fitted under a standard breast coil with a patient lying in the prone position. b) Image of the hardware compressing a gel phantom.

\subsection{Strain-Encoded (SENC)}

As introduced in [4], SENC is a method for directly measuring strain, which is defined as the percentage change in length of tissue given by $\varepsilon=\frac{\triangle L}{L_{0}}$, where $L_{0}$ is the initial tissue length and $\triangle L$ is the change in length due to tissue deformation. SENC imaging applies a tagging preparation pulse, which corresponds to a sinc peak at the tagging frequency, $\omega_{0}$. After deformation this peak shifts to different frequency. This shift, which is proportional to the strain, can be estimated by acquiring two images at two different z-encoding frequencies, $\omega_{L}$ and $\omega_{H}$. 

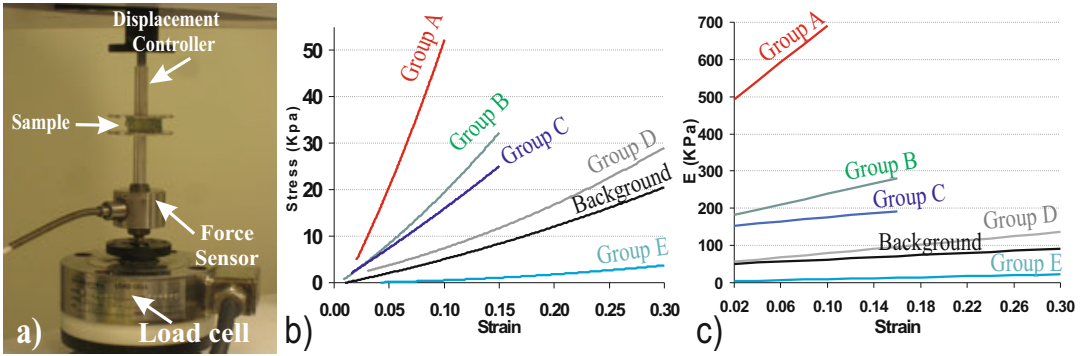

Fig. 2. a) Dynamic mechanical analyzer used to test each mass. b) Stress-strain curves for masses in Groups A, B, C, D, E and the background. c) Young's modulus (E) in KPa variation with strain for Groups A, B, C, D , E and the background.

\subsection{Phantom Composition}

In order to emulate breast cancer masses with different stiffnesses, a custom-made phantom was created by varying the $\mathrm{A}$ to $\mathrm{B}$ gel compounds mixing ratios of four different silicon materials. We constructed five groups of masses; masses of Groups A, B, C, and D were stiffer than the background, whereas masses of Group E were softer than the background. Table 1 shows the types of gel with corresponding mixing ratios. All of the masses were $10 \mathrm{~mm}$-thick cuboids, varying in plane from $3 \mathrm{~mm}$ to $12 \mathrm{~mm}$ (Fig.4 $4 \mathrm{a}, \mathrm{b}$ ).

We used a dynamic mechanical analyzer to determine the stiffness of the phantom (see Fig.2a). We used a ramped displacement to induce 10\%-30\% strain on the sample. Figure 2b shows non-linear stress-strain curves obtained from the dynamic mechanical analyzer, and Figure 2 s shows Young's modulus in KPa. Groups A, B, and C are respectively 8, 3, and 2 times stiffer than the background, while the stiffness of Groups $\mathrm{D}$ and $\mathrm{E}$ are very similar to the background. Comparing our phantom's Young's modulus with the ex-vivo breast stiffness measured by Samani et al. [3], masses of Group A would mimic malignant tumors, Groups B and C would mimic benign masses, while Groups D and E would mimic normal tissue. Table 1 shows Young's modulus for each gel mixtures calculated as: 1) the slope of a linear fit of the stress-strain data; 2) the differentiation of the second degree polynomial fit of the stress-strain data.

Table 1. Properties of different silicon masses, including mixing ratio, corresponding Young's modulus (E) in $\mathrm{KPa}$, and mass classification.

\begin{tabular}{|c|c|c|c|c|c|c|}
\hline Group & Material & Mixing ratio & \multicolumn{2}{|c|}{$\mathrm{E}(\mathrm{KPa})$} & Group:Back & Classification \\
\cline { 4 - 6 } & & $\mathrm{A}: \mathrm{B}$ & Linear fit & Polynomial fit & stiffness ratio & \\
\hline \hline $\mathrm{A}$ & $3-4207$ & $1.0: 1.0$ & 593 & $\mathrm{E}=2478 \varepsilon+443$ & $8.4: 1$ & Malignant \\
\hline $\mathrm{B}$ & $3-4133$ & $1.5: 1.0$ & 226 & $\mathrm{E}=714 \varepsilon+167$ & $3.2: 1$ & Benign \\
\hline $\mathrm{C}$ & $3-4207$ & $1.0: 1.2$ & 171 & $\mathrm{E}=278 \varepsilon+148$ & $2.4: 1$ & Benign \\
\hline D & $3-4133$ & $1.2: 1.0$ & 100 & $\mathrm{E}=284 \varepsilon+51$ & $1.4: 1$ & Normal \\
\hline Background & $\mathrm{A}-341$ & $1.0: 10.0$ & 71 & $\mathrm{E}=145 \varepsilon+48.5$ & - & Normal \\
\hline E & $3-4222$ & $1.0: 1.5$ & 20 & $\mathrm{E}=64 \varepsilon+2.9$ & $0.3: 1$ & Normal \\
\hline
\end{tabular}




\section{Experiments}

\subsection{Finite Element Method (FEM) Simulations}

To simulate the tissue deformation due to different compressions levels, we developed a finite element method using Matlab7.5®. We simulated a cross section of the phantom using $31 \times 55$ nodes connected together by two constant strain triangles having linear shape function. Fixed boundary conditions and Poisson ratio of 0.49 were assumed. For each compression level, we simulated a cross-section of the background containing five masses with $10 \mathrm{~mm}$ thick and width of 2, 4, 6, 8, and $10 \mathrm{~mm}$ (see Fig.3a). Multiple FEM iterations were performed to simulate the phantom's Young's modulus non-linearity.

\subsection{Scanning Protocol}

We performed scans on a 3T MRI Philips scanner (Achieva, Philips Medical Systems, Best, the Netherlands) using a four-channel phased array breast coil. All slices were scanned using in-plane resolution of $1 \times 1 \mathrm{~mm}^{2}$, field-of-view $=192 \times 192 \mathrm{~mm}^{2}$, slice thickness $=5 \mathrm{~mm}$. The phantom was scanned using: $\mathrm{T} 1$-weighted $(\mathrm{TR}=495 / \mathrm{TE}=10$ $\mathrm{ms}$, sense factor $=2)$, T2-weighted spin echo $(\mathrm{TR}=2500 / \mathrm{TE}=60 \mathrm{~ms}$, sense factor $=$ 2), SENC-CMP and SENC-REX protocols. For SENC scans we used ramp flip angles described in [7] with last flip angle $=80^{\circ}$, Tagging delay $=100 \mathrm{~ms}$, trigger delay $=500$ ms, segmented Cartesian K-space acquisition using turbo field echo (TFE) factor of 10 with no echo planar imaging (EPI), 19 compression cycles/slice.

For a given expected strain value at the boundaries of the compressing device, we used SENC equations given in [9] to calculate $\omega_{0}, \omega_{L}$, and $\omega_{H}$. In [9], Basha et al. showed that if the tissue of interest is compressed or stretched beyond the expected range, SENC measurements would saturate at either high or low strain values. Simulations showed that parts of the tissue would retain larger strain values than the strain applied at the tissue's boundaries; therefore, the scanning parameters were set to encompass strain values larger than levels applied at the boundaries (see Table2).

Table 2. Scanning parameters for measured strain values for SENC-CMP and SENC-REX scans at different levels of applied compression. All frequencies $\left(\omega_{0}, \omega_{L}, \omega_{H}\right)$ are in $\mathrm{mm}^{-1}$.

\begin{tabular}{|c|c|c|c||c|c|c|c|}
\hline \multicolumn{3}{|c||}{ SENC Compression (SENC-CMP) } & \multicolumn{4}{c|}{ SENC Relaxation (SENC-REX) } \\
\hline $\begin{array}{c}\text { Compression } \\
\text { at boundary }\end{array}$ & $\begin{array}{c}\text { Measured } \\
\text { strain range }\end{array}$ & $\omega_{0}=\omega_{L}$ & $\omega_{H}$ & $\begin{array}{c}\text { Relaxation } \\
\text { at boundary }\end{array}$ & $\begin{array}{c}\text { Measured } \\
\text { strain range }\end{array}$ & $\omega_{L}$ & $\omega_{0}=\omega_{H}$ \\
\hline \hline$-7 \%$ & 0 to $-10 \%$ & 1.8 & 2.0 & $+8 \%$ & 0 to $+10 \%$ & 2.0 & 2.2 \\
\hline$-10 \%$ & 0 to $-15 \%$ & 1.132 & 1.332 & $+11 \%$ & 0 to $+18 \%$ & 1.1111 & 1.3111 \\
\hline$-16 \%$ & 0 to $-22 \%$ & 0.708 & 0.908 & $+18 \%$ & 0 to $+25 \%$ & 0.8 & 1.0 \\
\hline$-23 \%$ & 0 to $-30 \%$ & 0.4667 & 0.6667 & $+29 \%$ & 0 to $+35 \%$ & 0.5714 & 0.7714 \\
\hline
\end{tabular}

\subsection{Quantification}

Masses were manually segmented and their detectability were quantified using the CNR Elastography defined by $\mathrm{CNR}_{e}=\frac{2\left(S_{\text {mass }}-S_{\text {background }}\right)^{2}}{\sigma_{\text {mass }}^{2}+\sigma_{\text {background }}^{2}}$, where $S$ and $\sigma$ are the mean and 


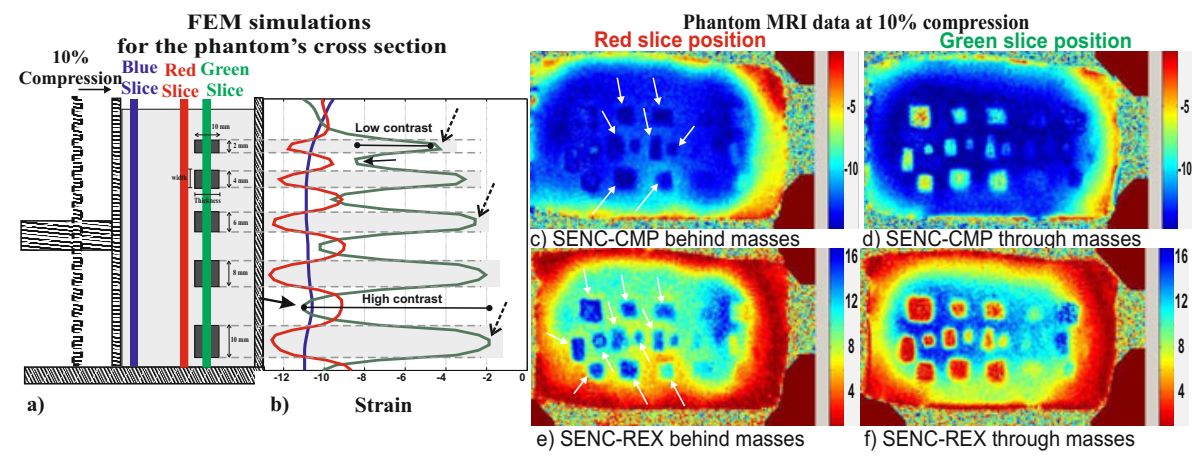

Fig. 3. a) FEM simulations while the phantom is compressed $10 \%$. b) Strain values at blue, red and green slices calculated from the FEM simulations. Solid black arrows point to different in background strain values depending on mass separation distances. Dotted black arrows point to different strain values within the masses depending on the mass width. c-f) Phantom MRI SENC-CMP and SENC-REX images. Slice positioned immediately behind the masses (c,e) and encompassing the masses $(\mathrm{d}, \mathrm{f})$ corresponds to the red and green slices in FEM simulations. White arrows point higher strain detected behind the masses.

standard deviation, respectively, and the background is a rectangular region surrounding the mass. Philips pride software was used to generate colored SENC strain images at the scanner console immediately after acquisition. For visual comparisons, all color pallets for SENC-CMP and SENC-REX were unified such that masses having low strain values are colored red, while normal background is colored blue.

\section{Results}

\subsection{FEM Simulation Results}

Figure 3 a shows a diagram for FEM simulations of a cross section of a homogeneous background with five different masses at $10 \%$ compression. All masses had same thickness $(10 \mathrm{~mm})$ but varied in width $(2,4,6,8$, and $10 \mathrm{~mm})$. Figure $3 \mathrm{~b}$ shows the corresponding $\mathrm{X}$-axis strain profile along blue, red, and green slices. The strain profile at the blue slice (furthest from the masses) remains constant around $-11 \%$, while the strain profile at the green slice (intersecting the masses) ranges from $-2 \%$ within the masses to $-11 \%$ in the surrounding background. By examining the simulation results, we observe the following:

1. The strain inside each masses depends on the width of the mass. The largest mass (10 $\mathrm{mm}$ in width) had strain of $-2 \%$, whereas the smallest mass ( $2 \mathrm{~mm}$ in width) had strain of $-4 \%$ (see dotted black arrows in Fig. $3 \mathrm{~b}$ ).

2. The strain measured within the background between the masses depends indirectly on the distance between the masses. If the separation between masses exceeds the combined width of the two masses, then the strain approaches the expected strain $(-11 \%)$. Conversely, if the separation between masses is less than the combined 

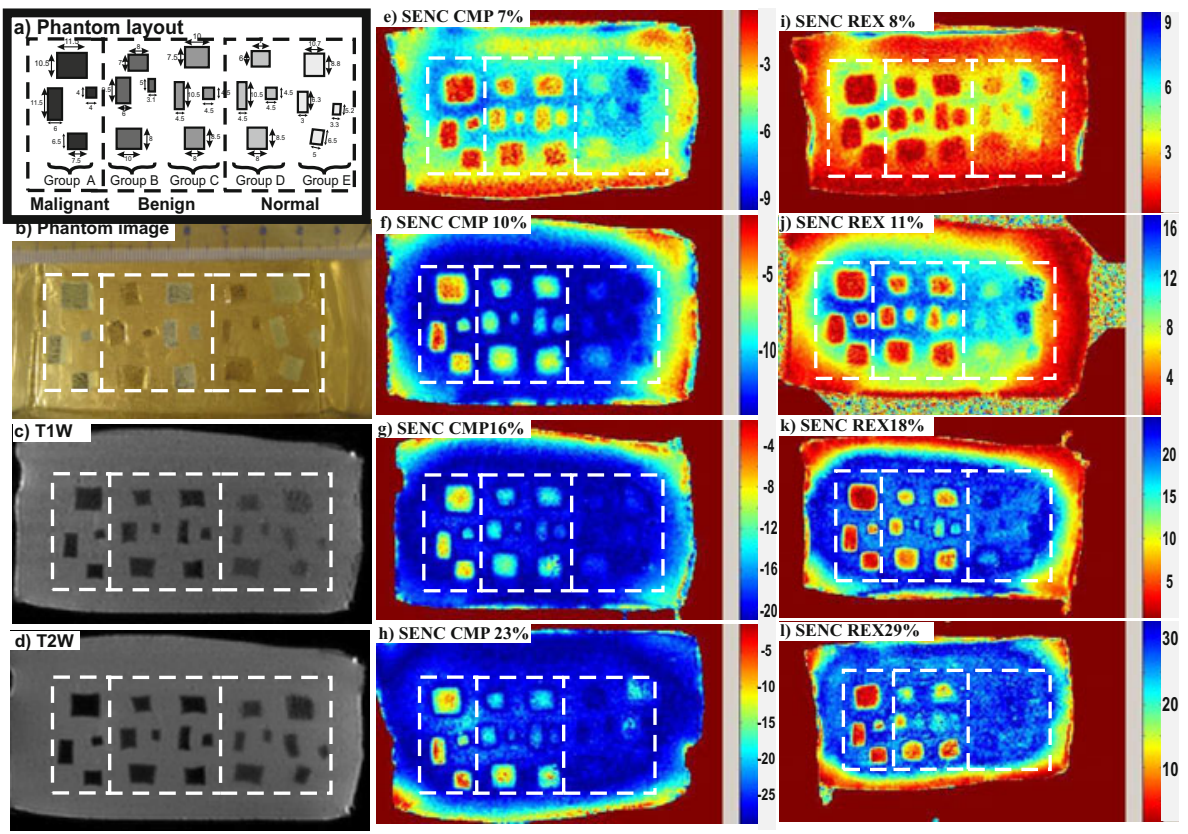

Fig. 4. a) Layout for the custom-made phantom containing five different groups of masses (dimensions are in $\mathrm{mm}$ ). b) Image showing masses during phantom manufacturing with ruler scale. Groups A, B, C, and D are stiffer than the background, while Group E is softer than the background. c) T1W image d) T2W image for the phantom. SENC-CMP images (e-h) and SENCREX images (i-l) for different compression levels and relaxation levels.

width of the masses, then the strain does not approach the expected strain (see solid black arrows in Fig.3b). This interaction between masses affects the $\mathrm{CNR}_{e}$ and consequently the ability to detect the masses.

3. The strain profile of the red slice (directly adjacent to the masses) appears to be mirroring the strain profile of the green slice (encompassing the masses). This is caused by the stiff masses pushing against the surrounding soft tissue (see Fig. 33).

\subsection{Phantom Results}

Figures 3 -f show SENC MRI images of the phantom at $10 \%$ compression. Figure $3 \mathrm{~d}$ and Figure 3f are SENC-CMP and SENC-REX images, respectively, at the same position as the green slice in the FEM simulations, which encompasses the masses. Both images confirm FEM simulations results, with nearly zero strain within the masses. Figure 3 and Figure 3 e show SENC-CMP and SENC-REX images, respectively, at the same position as the red slice in the FEM simulations, located adjacent to the masses. Arrows indicate the tissue surrounding the masses, which experience a higher level of compression than the applied external compression. This increases the confidence level of detecting the masses.

Figure $4 \mathrm{c}$ and Figure $4 \mathrm{~d}$ show $\mathrm{T} 1 \mathrm{~W}$ and $\mathrm{T} 2 \mathrm{~W}$ images of the phantom detecting all masses: malignant (Group A), benign (Groups B and C), and normal (Groups D and E). 
Table 3. Strain (mean \pm SD) measured from manually segmented masses form SENC-CMP and SENC-REX images at different compression and relaxation levels. Negative and positive signs indicates compression and relaxation, respectively.

\begin{tabular}{|c|c|c|c|c||c|c|c|c|}
\hline & \multicolumn{3}{|c|}{ SENC Compression (SENC-CMP) } & \multicolumn{3}{c|}{ SENC Relaxation (SENC-REX) } \\
\hline Compression & $-7 \%$ & $-10 \%$ & $-16 \%$ & $-23 \%$ & $+8 \%$ & $+11 \%$ & $+18 \%$ & $+29 \%$ \\
\hline \hline Group A & $-3.0 \pm 1.0$ & $-5.1 \pm 1.7$ & $-10.5 \pm 2.6$ & $-13.4 \pm 5.0$ & $1.1 \pm 0.8$ & $3.3 \pm 1.8$ & $5.5 \pm 4.3$ & $8.1 \pm 5.9$ \\
\hline Group B & $-3.7 \pm 0.9$ & $-8.2 \pm 1.3$ & $-14.5 \pm 2.0$ & $-18.1 \pm 4.5$ & $1.3 \pm 0.8$ & $4.5 \pm 1.9$ & $9.7 \pm 3.3$ & $15.2 \pm 5.5$ \\
\hline Group C & $-3.9 \pm 0.9$ & $-8.8 \pm 1.6$ & $-15.0 \pm 2.4$ & $-20.1 \pm 3.9$ & $1.4 \pm 0.8$ & $4.8 \pm 1.8$ & $10.4 \pm 3.6$ & $15.7 \pm 6.4$ \\
\hline
\end{tabular}

Figures 4 e-h show SENC-CMP images for compression levels of 7\%, 10\%, 16\%, and $23 \%$, respectively. Figures 4 -1 show SENC-REX images for relaxation levels of $8 \%$, $11 \%, 18 \%$, and $29 \%$, respectively. Malignant masses of Group A and benign masses of Groups B and C are easily detectable visually. However, normal masses of Groups D and $\mathrm{E}$ lacked enough contrast to distinguish them from the background.

With SENC-CMP images at 7\% compression and SENC-REX images at $8 \%$ and $11 \%$ compression, we could detect both malignant and benign but not normal masses; therefore, differentiating normal from suspicious masses. That said, these images had moderate $\mathrm{CNR}_{e}$ (11-20) and suffered from rim artifacts due to imperfect compressions. SENC-CMP images at 10\% compression and SENC-REX images at $18 \%$ had higher $\mathrm{CNR}_{e}$ of 60 and 30, respectively. This allowed the differentiation between malignant and benign masses (see Table 3 ).

By visual comparison, relaxation always performs better than compression. None of the SENC-CMP or SENC-REX images allowed differentiation between Group B and Group C, but these groups can be distinguished from Group A for all masses larger than $4 \times 4 \mathrm{~mm}^{2}$ using $16 \%$ and $23 \%$ compression levels as well as $18 \%$ and $29 \%$ relaxation levels. The smallest mass $\left(4 \times 4 \mathrm{~mm}^{2}\right)$ of Group A can sometimes be misclassified as Group B and C, which matches FEM simulation results described earlier.

\section{Discussion}

Our phantom results demonstrate that both malignant (Group A) and benign (Groups B and C) masses can be detected under all compression and relaxation levels. With small compression (7\%), SENC-CMP7\% image allows the detection of malignant and benign masses with moderate $\mathrm{CNR}_{e}(>10)$. The corresponding SENC-REX8\% image can also be used to detect all the masses, but has rim artifacts (see Fig.41). These artifacts are reduced when applying larger compression of 10\% resulting in SENC-REX11\% image, which has higher $\mathrm{CNR}_{e}$ and better detection capability. In order to differentiate between the benign and malignant masses we have to use even larger compression of $16 \%$ to obtain SENC-CMP16\% and SENC-REX18\% images.

In our phantom, the embedded masses were only eight times stiffer than the background. High-grade cancer masses are five to thirteen times stiffer than both fat and glandular tissue [3]; therefore, we would expect to see even more contrast in clinical trials. Our FEM simulations were limited to a cross-section of the phantom. If desired, this can be extended to 3D. Also, instead of using linear shape functions for the 
triangular elements, which results in a constant strain for each triangular element, we could use a more complicated model.

In conclusion, we have showed using FEM simulations and phantom study that SENC-CMP and SENC-REX are able to detect both benign- and malignant-like masses with compressions as small as $7 \%$. We have also shown that the slices adjacent to the masses show reverse contrast compared to slices containing the masses. These slices increase the confidence of our detection and classification method. Human trials need to be conducted in order to validate these phantom results. Normal volunteers, as well as patients having benign and malignant masses should be scanned. Therefore, we propose the following protocol for breast cancer screening: a) scan the whole breast using low compression. b) Examine SENC images at the scanner console using SENC on-line calculations to detect masses. c) If masses are detected, we would re-scan these slices using higher compression level in order to distinguish benign from malignant masses. Since higher compression would only be required for mass classifications, this protocol would maximize patient comfort without sacrificing detection and classification accuracy.

Acknowledgments. The authors would like to thank Shimon Unterman for his help with the stiffness measurements, as well as Issel Lim for her help in editing this paper. This work was supported in part by the following grants: NHLBI R01HL072704, NIH 1P50HL08946, 1R01CA100184, P50CA103175, P50CA88843, and U01CA140204.

\section{References}

1. American Cancer society, Cancer Facts and Figures 2009 (2009), http: //www . cancer . org

2. Bluemke, D.A., Gatsonis, C., Chen, M.H., DeAngelis, G.A., DeBruhl, N., Harms, S., Heywang-Kobrunner, S.H., Hylton, N., Kuhl, C.K., Lehman, C., et al.: Magnetic resonance imaging of the breast prior to biopsy. Jama 292(22), 2735 (2004)

3. Samani, A., Zubovits, J., Plewes, D.: Elastic moduli of normal and pathological human breast tissues: an inversion-technique-based investigation of 169 samples. Physics in Medicine and Biology 52(6), 1565-1576 (2007)

4. Osman, N., Sampath, S., Atalar, E., Prince, J.: Imaging longitudinal cardiac strain on shortaxis images using strain-encoded MRI. Magnetic Resonance in Medicine 46(2), 324-334 (2001)

5. Osman, N.: Detecting stiff masses using strain-encoded (SENC) imaging. Magnetic Resonance in Medicine 49(3), 606-608 (2003)

6. Fahmy, A., Krieger, A., Osman, N.: An integrated system for real-time detection of stiff masses with a single compression. IEEE Transactions on Biomedical Engineering 53(7), 1286-1293 (2006)

7. Harouni, A.A., Hossain, J., Jacobs, M.A., Osman, N.F.: Improved Hardware for Higher Spatial Resolution Strain-Encoded (SENC) MRI. Academic Radiology 18, 705-715 (2011)

8. Harouni, A.A., El Khouli, R.H., Hossain, J., Bluemke, D.A., Osman, N.F., Jacobs, M.A.: Enhancing mass detection and classification in breast tissue using Strain-ENCoded (SENC) breast MRI with histological validation. Submitted to Journal of Magnetic Resonance Imaging (March 2011)

9. Yousef, T.A., Osman, N.F.: Effect of Noise and Slice Profile on Strain Quantifications of Strain Encoding (SENC) MRI. In: Sachse, F.B., Seemann, G. (eds.) FIHM 2007. LNCS, vol. 4466, pp. 50-59. Springer, Heidelberg (2007) 